\title{
Overview of the Matrisome-An Inventory of Extracellular Matrix Constituents and Functions
}

\author{
Richard O. Hynes and Alexandra Naba \\ Howard Hughes Medical Institute, Koch Institute for Integrative Cancer Research, Massachusetts \\ Institute of Technology, Cambridge, Massachusetts 02139 \\ Correspondence: rohynes@mit.edu
}

Completion of genome sequences for many organisms allows a reasonably complete definition of the complement of extracellular matrix (ECM) proteins. In mammals this "core matrisome" comprises 300 proteins. In addition there are large numbers of ECMmodifying enzymes, ECM-binding growth factors, and other ECM-associated proteins. These different categories of ECM and ECM-associated proteins cooperate to assemble and remodel extracellular matrices and bind to cells through ECM receptors. Together with receptors for ECM-bound growth factors, they provide multiple inputs into cells to control survival, proliferation, differentiation, shape, polarity, and motility of cells. The evolution of ECM proteins was key in the transition to multicellularity, the arrangement of cells into tissue layers, and the elaboration of novel structures during vertebrate evolution. This key role of ECM is reflected in the diversity of ECM proteins and the modular domain structures of ECM proteins both allow their multiple interactions and, during evolution, development of novel protein architectures.

$T^{\mathrm{s}}$ he term extracellular matrix (ECM) means somewhat different things to different people (Hay 1981, 1991; Mecham 2011). Light and electron microscopy show that extracellular matrices are widespread in metazoa, underlying and surrounding many cells, and comprising distinct morphological arrangements. The initial biochemical studies on extracellular matrix concentrated on large, structural extracellular matrices such as cartilage and bone. In the 1980s, the availability of model systems such as the Engelbreth-Holm-Swarm (EHS) sarcoma opened the way to biochemical analyses of basement membranes and led to the discovery of the different group of ECM proteins that make up basement membranes. Biochemistry of native ECM was, and still is, impeded by the fact that the ECM is, by its very nature, insoluble and is frequently cross-linked. Furthermore, ECM proteins tend to be large, and early work was frequently on proteolytic fragments. The application of molecular biology to studies of ECM proteins and their genes uncovered many previously unknown ECM molecules and defined their structures. The protein chemistry and molecular biology revealed that ECM proteins are typically made up of repeated domains, often encoded in the genome as separate exonic units. The completion of the sequences of many genomes now

Editors: Richard O. Hynes and Kenneth M. Yamada

Additional Perspectives on Extracellular Matrix Biology available at www.cshperspectives.org

Copyright (C) 2012 Cold Spring Harbor Laboratory Press; all rights reserved; doi: 10.1101/cshperspect.a004903

Cite this article as Cold Spring Harb Perspect Biol 2012;4:a004903 
allows description of the entire list of proteins and, potentially, the definition of the complete repertoire of ECM proteins, based on homologies with known ECM proteins. Comparative analyses of the genomes of different organisms allow deductions about the evolution of this repertoire, which we term the matrisome. Newer methods such as mass spectrometry are also beginning to allow more detailed biochemical characterization of extracellular matrices. In this article, we will give an overview of the mammalian matrisome and briefly discuss certain aspects of the evolution of the matrisome and of the ECM.

\section{DEFINITION OF THE MATRISOME}

In analyzing the structure and functions of extracellular matrices, one would like to have a complete "parts list"—a list of all the proteins in any given matrix and a larger list of all the proteins that can contribute to matrices in different situations (the "matrisome"). As mentioned, the biochemistry of ECM is challenging because of the insolubility of most ECMs. However, the availability of complete genome sequences coupled with our accumulated knowledge about ECM proteins now makes it possible to come up with a reasonably complete list of ECM proteins. ECM proteins typically contain repeats of a characteristic set of domains (see figures and Table 1) (LamG, TSPN, FN3, VWA, Ig, EGF, collagen prodomains, etc.). Many of these domains are not unique to ECM proteins but their arrangements are highly characteristic. That is, the architecture of ECM proteins is diagnostic - they are built from assemblies of many ancient, and a few more recent, protein domains, each of which is typically encoded by one or a few exons in the genome. ECM proteins represent one of the earliest recognized and most elaborate examples of exon (domain) shuffling during evolution (Engel 1996; Patthy 1999; Hohenester and Engel 2002; Whittaker et al. 2006; Adams and Engel 2007). This characteristic of ECM proteins allows bioinformatic sweeps of the proteome encoded by any given genome, using a list of 50 or so domains to identify a list of candidate ECM proteins. Negative sweeps of that list using domains from other protein families (e.g, tyrosine kinases, which share FN3 and Ig domains with ECM proteins) and screens for transmembrane domains allow refinement of the list. A very few known ECM proteins do not have readily recognizable domains (e.g., elastin, dermatopontin, and some dentin matrix proteins) although, increasingly, even those are now being incorporated into protein analysis sites such as SMART and InterPro, allowing their routine capture in the sweeps. Using such methods plus manual annotation, we have been able to define a robust list of the proteins defining the mammalian matrisome by analysis of the human and mouse genomes (Naba et al. 2011). We call this list of "core" ECM proteins the core matrisome. It comprises $1 \%-1.5 \%$ of the mammalian proteome (without considering the contribution of alternatively spliced isoforms (prevalent in transcripts of matrisome genes). This list comprises almost 300 proteins, including 43 collagen subunits, three dozen or so proteoglycans, and around 200 glycoproteins.

This core matrisome list does not include mucins, secreted C-type lectins, galectins, semaphorins, and plexins and certain other groups of proteins that plausibly do associate with the ECM but are not commonly viewed as ECM proteins; lists of these "ECM-affiliated" proteins are given in Naba et al. (2011). The core matrisome list also does not include ECM-modifying enzymes, such as proteases, or enzymes involved in cross-linking, or growth factors and cytokines, although these are well known to bind to ECMs (see below).

Two useful databases provide information on the expression and distribution of various ECM proteins (http://www.matrixome.com/ bm/Home/home/home.asp, The Matrixome Project, maintained by Kiyotoshi Sekiguchi and http://www.proteinatlas.org/;Human Protein Atlas) (Ponten et al. 2008; Uhlen et al. 2010). A third database (MatrixDB, http:// matrixdb.ibcp.fr/) (Chautard et al. 2009, 2010) collates information about interactions among ECM proteins. 
The Matrisome-An Overview of ECM Constituents

\section{COLLAGENS}

Collagens are found in all metazoa and provide structural strength to all forms of extracellular matrices, including the strong fibers of tendons, the organic matrices of bones and cartilages, the laminar sheets of basement membranes, the viscous matrix of the vitreous humor, and the interstitial ECMs of the dermis and of capsules around organs. Collagens are typified by the presence of repeats of the triplet Gly-X-Y, where $\mathrm{X}$ is frequently proline and $\mathrm{Y}$ is frequently 4hydroxyproline. This repeating structure forms stable, rodlike, trimeric, coiled coils, which can be of varying lengths. A primordial collagen exon encoded six of these triplets (18 amino acids) encoded in 54 base pairs and, during evolution, this original motif has been duplicated, modified, and incorporated into many genes (Fig. 1A). Collagen subunits assemble as homotrimers or as restricted sets of heterotrimers and, in general, collagen subunits are very restricted in the partnerships they can form, although occasional promiscuity has been noted (for more details, see Ricard-Blum 2011; Yurchenco 2011).

Some of these genes are viewed as collagens, sensu stricto, whereas others that contain only short collagen segments are often referred to as "collagen-like" or "collagen-related." The distinction is to some extent arbitrary because many proteins viewed as "true" collagens also contain significant portions made up of other domains. The original type I collagen of bones and tendons consists almost entirely of a long $(\sim 1000$ amino acids) and rigid uninterrupted collagen triple helix (plus terminal noncollagenous prodomains that are removed during biosynthetic processing of the protein; Fig. 1A). The rodlike trimers assemble into higherorder oligomers and fibrils and become crosslinked by various enzymatic and nonenzymatic

\section{A}

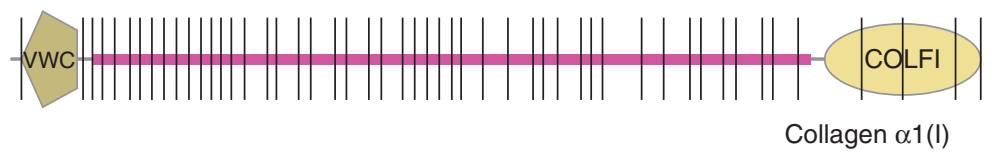

B

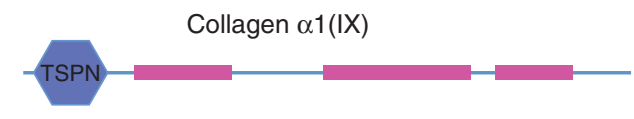

C

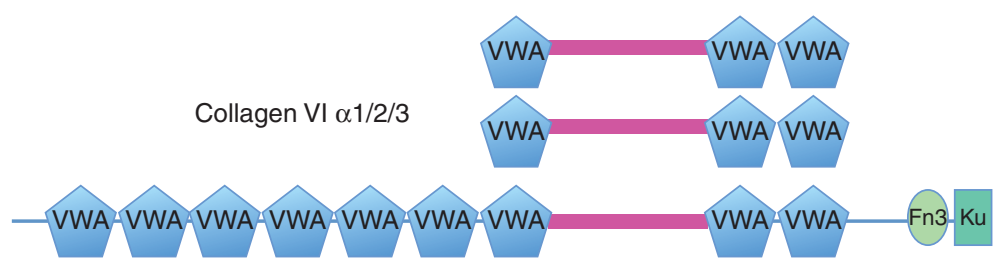

Figure 1. Examples of collagen structures. (A) Collagen I is a fibrillar collagen with a continuous collagen domain of around 1000 amino acids ( fuschia) comprising Gly-X-Y repeats that form a triple helix. It is encoded by multiple exons (note vertical lines) that are variants of a primordial exon encoding six such repeats. The collagen domain is flanked by amino- and carboxy-terminal noncollagenous domains that are removed by proteolysis to allow fibrillogenesis of the mature collagen. The VWC domain in this and other fibrillar collagens can be alternatively spliced and binds bone morphogenetic proteins (BMPs). (B) Collagen IX is a FACIT collagen (fibril-associated collagen with interrupted triple helix); the interruptions in the collagen domain allow bending. This and other FACIT collagens associate with fibrillar collagens and their amino-terminal domains extend out from the fibrils and presumably function as protein-binding domains. $(C)$ Collagen VI is a heterotrimer of three related subunits, one of which is much longer and forms globular heads at each end. VWA domains are commonly protein-binding domains and probably allow interactions with other proteins during the formation of short fibrils by collagen VI. 
reactions conferring considerable structural strength. Several other collagens with similar fibrillar structure are found in various tissues. Many other collagen types have interruptions in the Gly-X-Y repeating structure, introducing flexibility into the molecules. All collagen genes also encode additional noncollagenous domains, some of which are the characteristic collagen $\mathrm{N}$ and $\mathrm{C}$ prodomains, whereas others are domains shared with other ECM proteins and retained in the mature proteins (Fig. $1 \mathrm{~B}, \mathrm{C})$. These additional protein domains confer specific binding affinities, allowing collagen molecules to interact with each other and with other proteins to assemble the various structures. The diversity of collagen structures, genes, and assemblies is discussed by Ricard-Blum (2011) and the assembly of type IV collagen into the laminar structure of basement membranes is reviewed by Yurchenco (2011). Other reviews of the collagen family cover additional aspects (Eyre and Wu 2005; Robins 2007; Gordon and Hahn 2009).

Among the collagen-like or collagen-related proteins (see table in Ricard-Blum 2011), a few are membrane proteins; others, such as complement component $\mathrm{Clq}$ and related proteins are secreted but their main functions do not involve ECM and they are not considered as part of the ECM or matrisome; yet others, such as the collagen-like domain of acetylcholinesterase, serve to anchor other proteins into the ECM, and some, such as EMIDs, are true ECM proteins. It is worth keeping in mind the possibility that the presence of collagen-like domains could act to bind some of these non-ECM proteins to the ECM, at least part of the time; in that sense they are ECM-associated.

\section{PROTEOGLYCANS}

Proteoglycans are interspersed among the collagen fibrils in different ECMs. Rather than providing structural strength, they confer additional properties. Proteoglycans are glycoproteins with attached glycosaminoglycans (GAGs; repeating polymers of disaccharides with carboxyl and sulfate groups appended). The addition of GAGs confers on proteoglycans a high negative charge, leading them to be extended in conformation and able to sequester both water and divalent cations such as calcium. These properties confer space-filling and lubrication functions. GAGs, especially heparan sulfates, also bind many secreted and growth factors into the ECM (see Sarrazin et al. 2011 for more details).

There are around three dozen extracellular matrix proteoglycans encoded in mammalian genomes; they fall into several families (Table 1) (see also Iozzo and Murdoch 1996). The two largest are those based on LRR repeats (Merline et al. 2009; Schaefer and Schaefer 2010) and those containing LINK and C-type lectin domains (hyalectans). Many of the LRR proteoglycans bind to various collagens and to growth factors and the hyalectan family members bind to various ECM glycoproteins such as tenascins, and through the LINK domain, to hyaluronic acid. These binding functions contribute to regulation of protein complexes in the ECM.

In addition, there are around a dozen proteoglycans that do not fall into these two families (e.g., lubricin/PRG4, endocan/ESM1, serglycin, and three testicans related to SPARC/osteonectin; see Table 1). Perhaps the most significant of all is perlecan (HSPG2), a multidomain protein that is a core proteoglycan of all basement membranes (see Table 1 and below). There are also many examples of proteins falling into other categories (e.g., some collagens, agrin, betaglycan, CD44, and other glycoproteins) that are sometimes or always modified by attachment of GAGs, which could lead one to consider them also as proteoglycans. The boundary between proteoglycans and glycoproteins is thus somewhat a matter of definition. The consensus view is to consider as proteoglycans those that have a significant fraction of their total mass made up by GAGs.

There are also two small families of integral membrane proteoglycans: glypicans (Filmus et al. 2008) and syndecans (Couchman 2010; Xian et al. 2010), both of which bear heparan sulfate side chains as does CD44, and there are a few additional transmembrane chondroitin sulfate proteoglycans. Further details of structure and functions of various heparan sulfate 
Table 1. Extracellular matrix proteoglycans

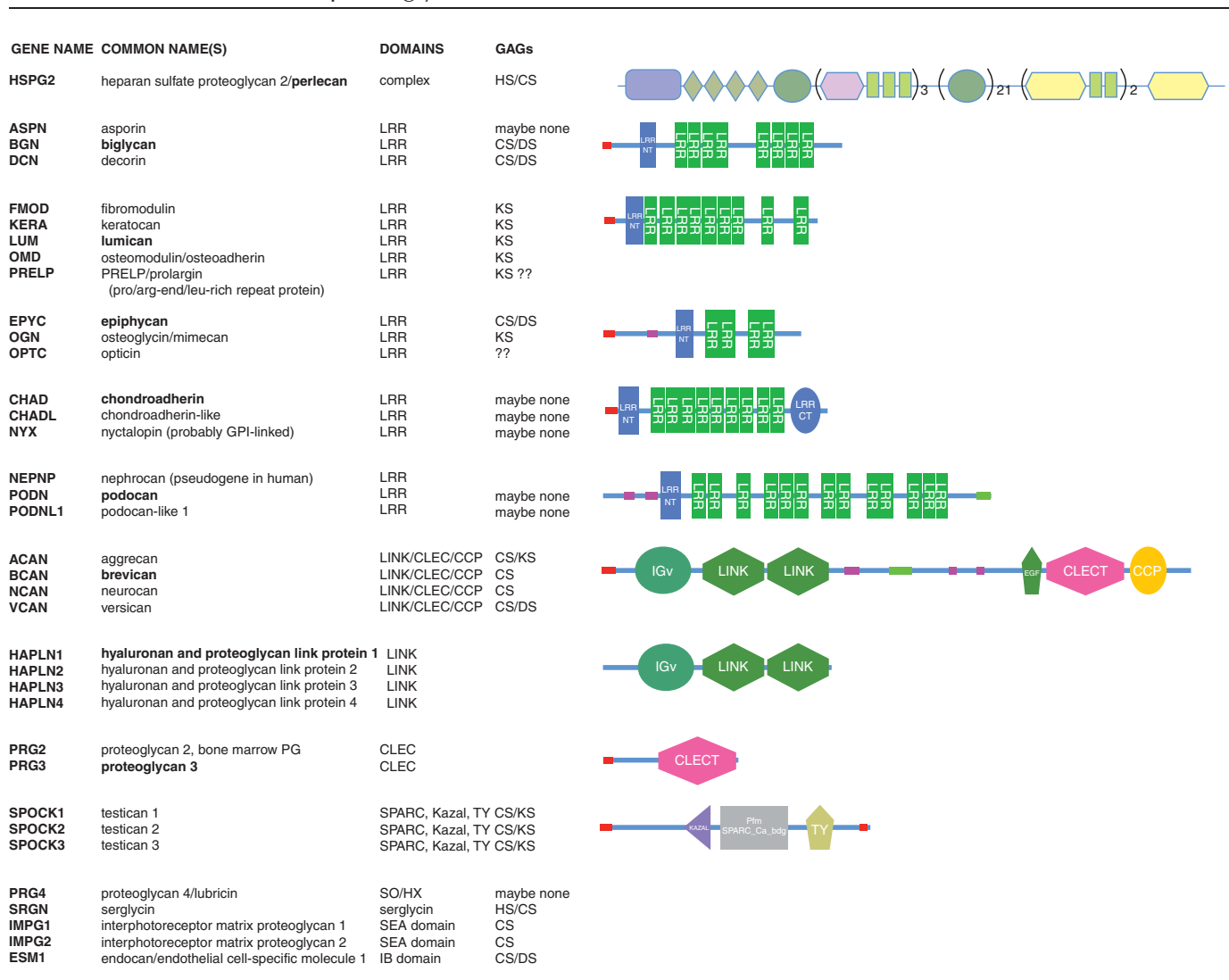

Table lists known ECM proteoglycans and shows representative domain structures.

There are five subfamilies of small leucine-rich repeat (LRR) proteoglycans based on sequence homologies (Merline et al. 2009; Schaefer and Schaefer 2010) —A representative structure is shown for one member (bold) from each subfamily. LRR repeat regions in other proteins are involved in protein-protein interactions.

The hyalectans (aggrecan, brevican, neurocan, and versican) all share a similar structure with Ig-LINK-LINK at their amino terminus and EGF(1 or 2)-CLEC-CCP at their carboxyl terminus with variable lengths of sequence between these two sets of domains. The central intervening sequence bears the GAG side chains and is almost 2000 amino acids long in aggrecan, and in versican it varies from a few to almost 2700 amino acids through alternative splicing. The LINK domains in both hyalectans and in the four link proteins bind to hyaluronan with nanomolar affinity and the EGF-CLEC-CCP cluster, which is similar to that in selectins, binds carbohydrates.

The domain structures were extracted from the SMART database (http://smart.embl-heidelberg.de). The domain structure of perlecan, the major proteoglycan of basement membranes, includes multiple domains in N-C order (SEA, LDLa, Ig, LamB, EGF, Ig, LamG, EGF). The glycosaminoglycan (GAG) content of each proteoglycan is based on the reviews cited above (HS, heparan sulfate; CS, chondroitin sulfate; DS, dermatan sulfate; KS, keratan sulfate).

proteoglycans are discussed by Bishop et al. (2007) and Sarrazin et al. (2011).

\section{GLYCOPROTEINS}

In addition to the collagens and proteoglycans that provide strength and space-filling functions (among others), there are around 200 complex glycoproteins in the mammalian matrisome (see Table 2 and Naba et al. 2011). These confer myriad functions including interactions allowing ECM assembly, domains and motifs promoting cell adhesion, and also signaling into cells and other domains that bind growth factors. The bound growth factors can serve as reservoirs that can be released (e.g., by proteolysis) 
R.O. Hynes and A. Naba

Table 2. Extracellular matrix glycoproteins

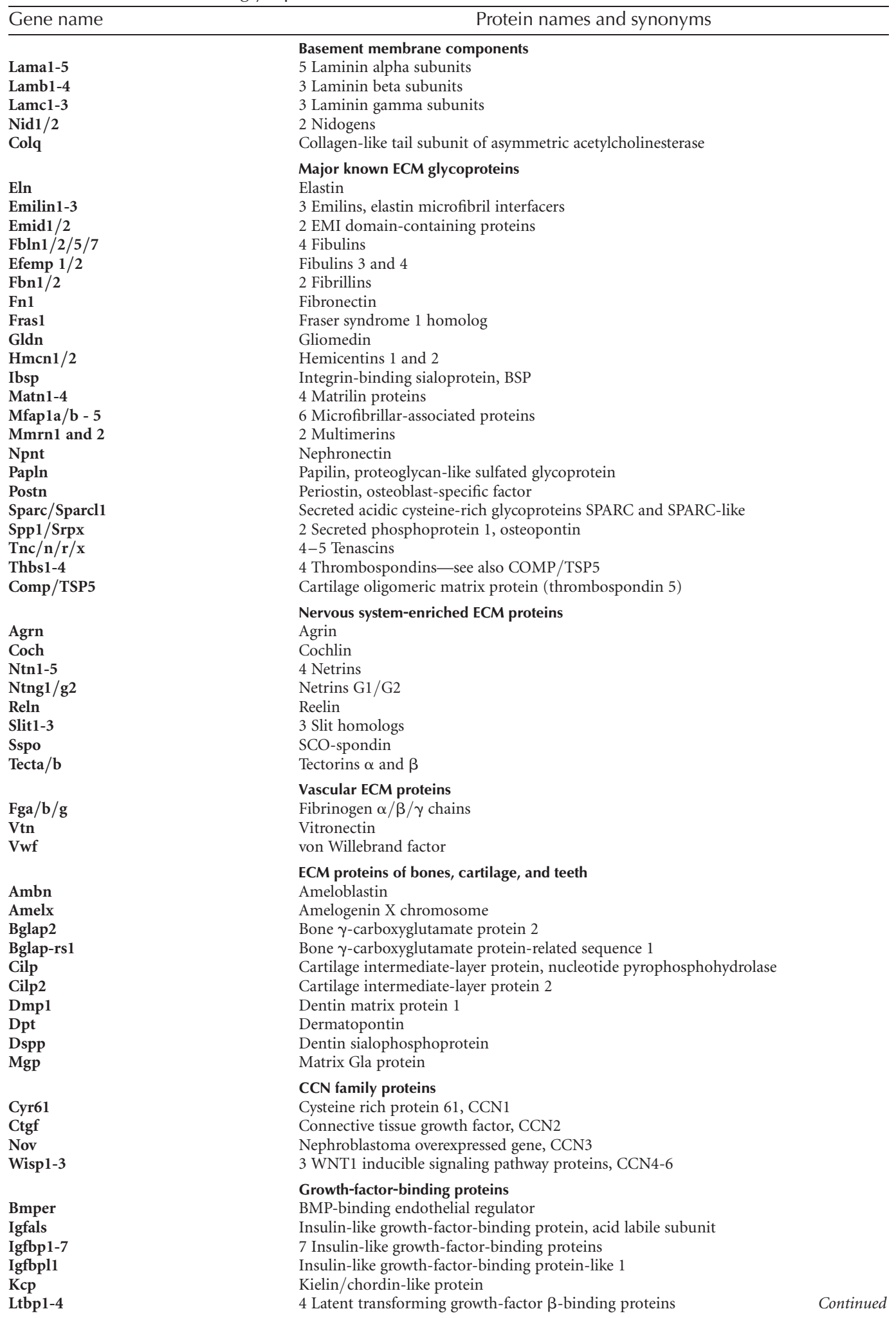


Table 2. Continued

\begin{tabular}{|c|c|}
\hline Gene name & Protein names and synonyms \\
\hline & Other possible ECM proteins \\
\hline Abi3bp & ABI gene family, member $3(\mathrm{NESH})$-binding protein \\
\hline Adipoq & Adiponectin, $\mathrm{C} 1 \mathrm{Q}$, and collagen domain-containing protein \\
\hline Aebp1 & AE-binding protein 1 \\
\hline Bsph1 & Binder of sperm protein homolog 1 \\
\hline Cdcp2 & CUB domain-containing protein 2 \\
\hline Creld1/2 & Cysteine-rich with EGF-like domains 1 and 2 \\
\hline Crim1 & Cysteine-rich transmembrane BMP regulator 1 (chordin like) \\
\hline Crispld1/2 & Cysteine-rich secretory protein LCCL domain-containing 1 and 2 \\
\hline Cthrc1 & Collagen triple helix repeat containing 1 \\
\hline Ddx26b & DEAD/H (Asp-Glu-Ala-Asp/His) box polypeptide 26B \\
\hline Dmbt1 & Deleted in malignant brain tumors 1 \\
\hline Ecml & Extracellular matrix protein 1 \\
\hline Ecm2 & Extracellular matrix protein 2 , female organ, and adipocyte specific \\
\hline Edil3 & EGF-like repeats and discoidin I-like domains 3 \\
\hline Egflam & EGF-like, fibronectin type III, and laminin G domains \\
\hline Fgl1 $/ 2$ & Fibrinogen-like proteins 1 and 2 \\
\hline Fndc1 $/ 7 / 8$ & 3 Fibronectin type III domain-containing proteins \\
\hline Gas6 & Growth arrest specific 6 \\
\hline Igsf10 & Immunoglobulin superfamily, member 10 \\
\hline Lgil-4 & 4 Leucine-rich repeat LGI family proteins \\
\hline Lrg1 & Leucine-rich $\alpha$-2-glycoprotein 1 \\
\hline Mepe & Matrix extracellular phosphoglycoprotein with ASARM motif \\
\hline Mfge8 & Milk fat globule-EGF factor 8 protein \\
\hline Nell1/2 & NEL-like 1 and 2 \\
\hline Oit3 & Oncoprotein-induced transcript 3 \\
\hline Otog & Otogelin \\
\hline Pcolce/Pcolce2 & 2 Procollagen C-endopeptidase enhancer proteins \\
\hline Pxdn & Peroxidasin homolog \\
\hline Rspo1-4 & 4 R-spondin homologs \\
\hline Slamf6 & SLAM family member 6 \\
\hline Smoc1 & SPARC-related modular calcium-binding proteins 1 and 2 \\
\hline Sned1 & Sushi, nidogen, and EGF-like domains 1 \\
\hline Spon1 & Spondin 1, (f-spondin) extracellular matrix protein \\
\hline Spon2 & Spondin 2, extracellular matrix protein \\
\hline Srpx2 & Sushi-repeat-containing protein, $\mathrm{X}$-linked 2 \\
\hline Svep1 & Sushi, von Willebrand factor type A, EGF, and pentraxin domain-containing 1 \\
\hline Tgfbi & Transforming growth factor $\beta$-induced \\
\hline Thsd4 & Thrombospondin type I domain-containing 4 \\
\hline Tinag/Tinagl1 & Tubulointerstitial nephritis antigen/tubulointerstitial nephritis antigen-like 1 \\
\hline Tnfaip6 & Tumor necrosis factor $\alpha$-induced protein 6 \\
\hline Tsku & Tsukushin \\
\hline Vit & Vitrin \\
\hline Vwa1-5 & 5 von Willebrand factor A domain-containing proteins \\
\hline $\mathrm{Vwc} 2 / 2 \mathrm{l} / \mathrm{e}$ & 3 von Willebrand factor $\mathrm{C}$ domain-containing proteins \\
\hline $\mathrm{VwD} / \mathrm{E}$ & 2 von Willebrand factor $\mathrm{D}$ and EGF domain proteins \\
\hline Zp1-4 & 4 Zona pellucida glycoproteins \\
\hline Zp3r & Zona pellucida 3 receptor \\
\hline \multirow[t]{2}{*}{ Zpld1 } & Zona pellucida-like domain-containing 1 \\
\hline & Novel predicted ECM proteins \\
\hline 2010321M09Rik & RIKEN cDNA 2010321M09 gene \\
\hline 5430419D17Rik & RIKEN cDNA 5430419D17 gene \\
\hline 6130401L20Rik & RIKEN cDNA 6130401L20 gene \\
\hline 9230107M04Rik & RIKEN cDNA 9230107M04 gene \\
\hline A930038C07Rik & RIKEN cDNA A930038C07 gene \\
\hline AW551984 & Expressed sequence AW551984 \\
\hline C330046G03Rik & RIKEN cDNA C330046G03 gene \\
\hline D17H6S56E-3 & DNA segment, Chr 17, human D6S56E 3 \\
\hline Gm106 & Predicted gene 106 \\
\hline Gm414 & Predicted gene 414 \\
\hline Gm6924 & Predicted gene 6924 \\
\hline
\end{tabular}

Table lists known and predicted ECM glycoproteins other than collagens and proteoglycans, defined largely by their characteristic domain content. The table is split into rough categories-individual glycoproteins are listed in only a single category although many could be listed under more than one. The first page lists glycoproteins about which a significant amount is known; the second page lists glycoproteins about which relatively little to nothing has been reported concerning their role in the ECM. 
or can be presented as solid-phase ligands by the ECM proteins (Hynes 2009).

The best-studied ECM glycoproteins are the laminins ( 11 genes; $5 \alpha, 3 \beta, 3 \gamma$ ) and fibronectins (1 gene encoding multiple splice isoforms). These are reviewed in detail by Aumailley et al. (2005) and Yurchenco (2011) and by Schwarzbauer and DeSimone (2011), respectively. Also well studied are the thrombospondins and tenascins, reviewed by Bentley and Adams (2010) and Adams and Lawler (2011) and by Chiquet-Ehrismann and Turner (2011), respectively. The structures of these glycoproteins are well known and exemplify the typical multiple repeating domain structure and extended multimeric forms of ECM proteins (Fig. 2). The same is true for fibulins (de Vega et al. 2009) and nidogens (Ho et al. 2008; Yurchenco 2011) and many others. Two subgroups of ECM glycoproteins have been studied particularly in the context of the nervous system (netrins, slits, reelin, agrin, SCO-spondin-see article by Barros et al. [2011] and Fig. 3) and the hemostatic system (von Willebrand factor, vitronectin, and fibrinogen-a facultative ECM protein) (Bergmeier et al. 2008; Bergmeier and Hynes 2011). These two biological systems also involve roles for more widely distributed ECM proteins such as thrombospondins, fibronectins, laminins, collagens, proteoglycans, etc. Similarly, the matrices of other tissues typically contain both ubiquitous and tissue-restricted ECM glycoproteins. Another group of ECM glycoproteins that has been studied in the context of disease and the regulation of transforming growth factor beta (TGF- $\beta$ ) functions includes the fibrillins and LTBPs (Ramirez and Dietz 2009; Ramirez and Rifkin 2009; see article by Munger and Sheppard 2011).

However, as can be seen in Table 2, there are multiple other ECM glycoproteins about which much less (in some cases, almost nothing) is known. These include some enormous glycoproteins with impressive arrays of domains, such as SCO-spondin (59 domains of seven types) and hemicentin-1, also known as fibulin-6 (61 domains of six types), and many that are affected in disease (Aszódi et al. 2006; Nelson and Bissell 2006; Bateman et al. 2009). It will be of considerable interest to learn the distributions and functions of this diverse set of ECM glycoproteins and we can expect that the approaches that have been effective for the better-studied proteins will provide many insights into the roles of those less well known and novel.

\section{ECM-BOUND GROWTH AND SECRETED FACTORS}

As mentioned above and elsewhere (Hynes 2009; Ramirez and Rifkin 2009; Rozario and DeSimone 2010), many growth factors bind to ECM proteins and must be considered also as constituents of extracellular matrices. One popular idea is that growth and other secreted factors bind to GAGs, especially heparan sulfates. Although this is undoubtedly true, there are clear examples of growth factors binding to specific domains of ECM proteins. Fibronectin binds specifically to a variety of growth factors (VEGF, HGF, PDGF, etc.; Rahman et al. 2005; Wijelath et al. 2006; Lin et al. 2010) and the VWC/chordin and follistatin domains found in many ECM proteins (see Figs. 1-3) are known to bind BMPs (Wang et al. 2008; Banyai et al. 2010). TGF binds specifically to TB domains in LTBPs, which bind in turn to fibrillins and to fibronectin-rich matrices (Ramirez and Rifkin 2009; Munger and Sheppard 2011). These ECM-TGF interactions have significant consequences for genetic diseases; mutations in fibrillins affect the regulation of TGF- $\beta$ function in Marfan's syndrome and in other diseases (Ramirez and Dietz 2009).

It seems virtually certain that the known examples of growth factor binding to ECM, including directly to ECM proteins, presage many more such cases, and this aspect of ECM function is in great need of further investigation. The ECM can act as a reservoir or sink of such factors and there are many examples of this for chemokines and for many of the most important developmental signals (e.g., VEGFs, Wnts, Hhs, BMPs, and FGFs). Such factors form gradients that control pattern formation during developmental processes and it is clear that some of those gradients are markedly 


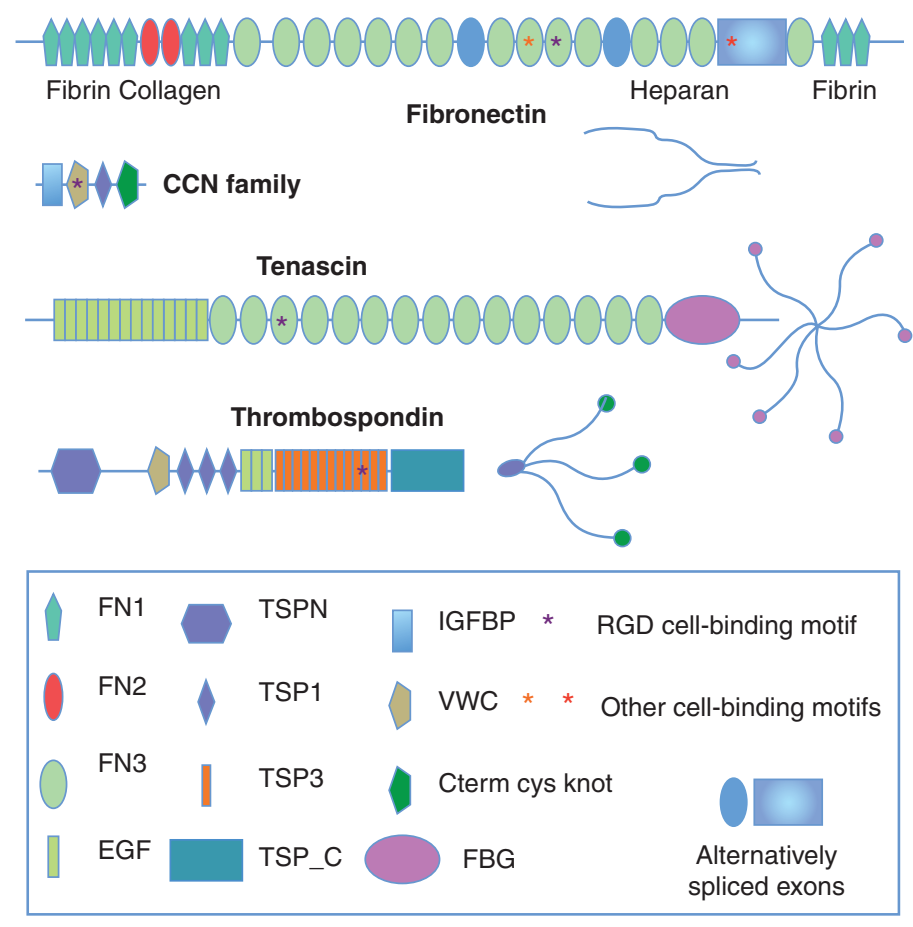

Figure 2. Examples of characteristic ECM glycoprotein structures. Note the multidomain structure of these ECM glycoproteins. Each domain is typically encoded by a single exon or a small set of exons. This has allowed shuffling of domains into different combinations during evolution. Individual domains are specialized for binding different proteins, as indicated for fibronectin. Some domains are alternatively spliced, as noted for fibronectin, and is also true for tenascin (not shown). Cell-binding motifs such as RGD and LGV are indicated by asterisks. Fibronectin dimerizes through disulfide bonding at the carboxyl terminus, whereas thrombospondin and tenascin form trimers and hexamers, respectively, through coiled coil domains and disulfide bonds near the amino terminus. The appearances of the intact protein multimers (as would be seen by electron microscopy) are diagrammed. Note that growth factor-binding domains (IGFBP, VWC, and others) are included in many ECM proteins. CYR61 is shown as a representative member of the CCN family (see Table 2), small ECM proteins that contain integrin-binding motifs and growth-factor-binding domains (IGFBP and VWC) and are known to regulate growth factor functions (Chen and Lau 2009) as are the larger proteins shown.

affected by ECM binding (Yan and Lin 2009). Indeed, it seems probable that many more gradients incorporate ECM binding as part of their regulation. Investigation of this concept will be greatly aided by our current fairly complete inventory of ECM proteins and their constituent domains.

\section{MODIFIERS OF ECM STRUCTURE AND FUNCTION}

Another aspect of ECM function is that ECM proteins and the fibrils into which they assemble are subsequently often significantly modified.
Collagens have long been known to become cross-linked by disulfide bonding, transglutaminase cross-linking, and through the action of lysyl oxidases and hydroxylases (Eyre and Wu 2005; Robins 2007; Ricard-Blum 2011). Laminins and other basement membrane proteins also become cross-linked by disulfide bonding (see Yurchenco 2011 for further details) and the same is true of fibronectin, which also undergoes further processing to a state characterized by insolubility in deoxycholate (DOC) (Choi and Hynes 1979; Schwarzbauer and DeSimone 2011). The exact basis for this insolubility is not known, but 
R.O. Hynes and A. Naba
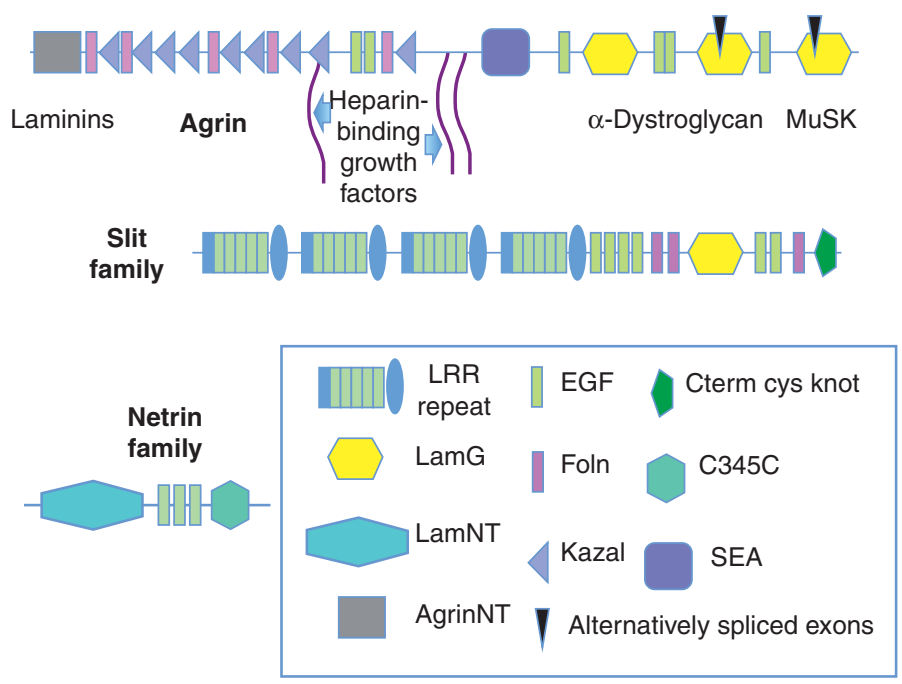

Figure 3. Glycoproteins with special roles in the nervous system. These three proteins are involved in synapse formation (Agrin) and in axonal guidance (Slits and Netrins). Sites for binding other ECM proteins (laminins), growth factors, and cell-surface receptors ( $\alpha$-dystroglycan and MuSK) are indicated for agrin. Slit also contains known GF-binding domains (Foln). Agrin has two small alternatively spliced exons that markedly affect its functions, a characteristic of many ECM proteins. Unlike many ECM proteins, the major receptors for these three proteins are not integrins. Slit family proteins bind to Robo receptors, whereas Netrins bind to Unc5 and DCC receptors. The functions of these ECM-receptor pairs in the CNS are discussed in Barros et al. (2011) but they also function in other aspects of cell and tissue pattern regulation during development. They are evolutionarily ancient and are conserved in all bilaterial phyla.

fibronectin and other ECM proteins are also substrates for transglutaminase 2, which undoubtedly contributes to the insolubility of ECM (Lorand and Graham 2003; Iismaa et al. 2009).

Proteolytic enzymes also modify the ECMindeed, procollagen propeptidases are necessary to process collagens so that they can polymerize. Collagens and other ECM proteins are also substrates for matrix metalloproteases (MMPs) (Page-McCaw et al. 2007; Cawston and Young 2010), ADAMs (Murphy 2008) and ADAMTS proteases (Porter et al. 2005; Apte 2009), and many other proteolytic enzymes (elastases, cathepsins, various serine esterase proteases, etc.) can also act on many ECM proteins (see article by Lu et al. 2011). These various proteolytic processes play roles in ECM turnover and are thought to release ECM-bound growth factors and also to expose cryptic activities in the ECM (Mott and Werb 2004; Ricard-Blum 2011), including the release of antiangiogenic inhibitors (Bix and Iozzo 2005; Nyberg et al. 2005; Hynes 2007). Similarly, enzymes that degrade GAGs, such as heparanases and sulfatases, can also alter the properties of ECM proteoglycans (see articles by $\mathrm{Lu}$ et al. 2011; Sarrazin et al. 2011). The remodeling of ECM by these various processes has major effects on development and pathology (Daley et al. 2008; Kessenbrock et al. 2010; $\mathrm{Lu}$ et al. 2011). Lists of these ECM-modifying enzymes can be found in the reviews cited and in Naba et al. (2011).

\section{CELLULAR RECEPTORS FOR EXTRACELLULAR MATRIX}

For the ECM to affect cellular functions, it is obvious that there must be receptors for ECM proteins. The major receptors are the integrin family, comprising $24 \alpha \beta$ heterodimers (Fig. 4). These have been extensively reviewed elsewhere and specific aspects are covered in 


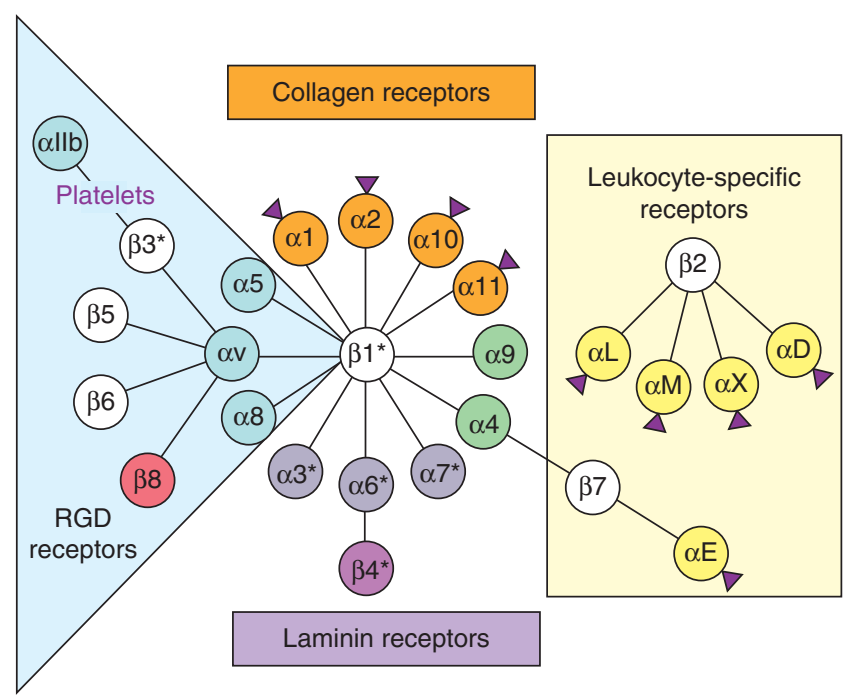

Figure 4. Integrin receptors for ECM proteins. The diversity of integrin subunits and their interactions. Shown are the mammalian integrins, separated by color coding into subsets of closely related subunits. The RGD- binding (blue) and laminin-binding (purple) subclasses are evolutionarily very ancient and found in all metazoan phyla, but they have diverged into clades in the vertebrate lineage. The $\alpha 4 / \alpha 9$ clade (green) is vertebrate-specific. Two subclasses of chordate $\alpha$ subunits have inserted I domains (purple arrowheads); they include collagen-specific integrins (orange) and a set of $\alpha$ subunits confined to leukocytes (yellow). Some subunits show alternatively spliced isoforms $\left({ }^{*}\right)$. The leukocyte integrins bind predominantly to cell-surface counterreceptors, whereas integrins containing either the $\beta 1$ or $\alpha \mathrm{v}$ subunits bind predominantly to extracellular matrix (ECM) proteins, although within each class there are exceptions to these generalizations and it is worth noting that most integrins are capable of binding multiple ligands, and there are many others beyond those shown here (Humphries et al. 2006). Many, if not all, $\alpha \mathrm{v}$ integrins are also capable of activating TGF- $\beta$. Most $\beta$ subunits are highly related (white) and bind to talin and related proteins (Campbell and Humphries 2011; Wickström et al. 2011 ), whereas the $\beta 4$ subunit instead binds to intermediate filaments through specific linker proteins and the $\beta 8$ subunit binds to band 4.1 proteins instead of to talin. (figure modified from Hynes 2002).

other articles in this collection (Schwartz 2010; Campbell and Humphries 2011; Geiger and Yamada 2011; Huttenlocher and Horwitz 2011; Watt and Fujimura 2011; Wickström et al. 2011). Another receptor for ECM proteins is dystroglycan, which binds to laminin, agrin, and perlecan in basement membranes as well as to the transmembrane neurexins (Barresi and Campbell 2006). Each of these dystroglycan ligands contains LamG domains, which bind to dystroglycan in a glycosylation-dependent manner (see Fig. 3), probably by binding carbohydrate side chains on dystroglycan. Mutations in dystroglycan or its associated proteins in the membrane or the cytoskeleton (or in laminin) can all produce various forms of muscular dystrophy, because of the loss of the transmembrane connection to the basement membrane surrounding the muscle cells. Other cellular receptors for ECM include GPVI on platelets and the DDR (discoidin domain receptor) tyrosine-kinases, all of which are receptors for collagens (Leitinger and Hohenester 2007), the GPIb/V/IX complex, which forms a receptor for von Willebrand factor on platelets (Bergmeier et al. 2008; Bergmeier and Hynes 2011), and CD44, which binds to hyaluronan and is expressed on many cells. As noted in Figure 3, Slits bind to Robo receptors of the Ig superfamily and netrins bind to Unc5-related tyrosinekinase receptors or to DCC, an Ig superfamily receptor, whereas agrin binds to the MuSK tyrosine-kinase receptor. Thus, although integrins comprise the dominant class of ECM 
receptors and are present on most cells, numerous other receptors for ECM proteins are expressed on specific cell types.

In addition to binding extracellular ligands, these ECM receptors provide transmembrane links to the cytoskeleton and to signal transduction pathways. The cytoplasmic domains of ECM receptors assemble large and dynamic complexes of proteins, which regulate cytoskeletal assembly and activate many signaling cascades within cells (Geiger and Yamada 2011). In the case of integrins, these submembranous complexes also regulate the extracellular affinity of the receptors (so-called "inside-out" signaling) and the same may be true of other classes of ECM receptors. It has become clear that the signaling functions of ECM adhesion receptors are at least as complicated as those of canonical growth factor receptors and that engagement of ECM receptors provides signals regulating cellular survival, proliferation, and differentiation as well as adhesive and physical connections involved in cell shape, organization, polarity, and motility.

\section{EVOLUTION OF THE MATRISOME AND THE EXTRACELLULAR MATRIX}

The $\sim 300$ proteins that make up the core matrisome in mammals are a mixture of very ancient proteins and some much newer ones (Fig. 5). Comparative analyses of the genomes of different taxa have revealed that some ECM proteins are shared by almost all metazoa, even simple organisms such as sponges, coelenterates, and cnidaria (Huxley-Jones et al. 2007; Ozbek et al. 2010). Most notable are the proteins that make up the core of basement membranestype IV collagens ( 2 subunits), laminin (4 genes, $2 \alpha, 1 \beta$, and $1 \gamma$ ), nidogen, and perlecan (1 gene each)—see Yurchenco 2011. We call this set of genes the basement membrane toolkit and it is found in all protostome and deuterostome genomes and must therefore have been present in the common ancestor of all bilateria (Hynes and Zhao 2000; Whittaker et al. 2006). Many, but not all, of these genes are also found in more primitive metazoan organisms such as cnidaria and sponges (Putnam et al. 2007;
Chapman et al. 2010; Srivasatava et al. 2010). It is plausible to argue that the evolution of multilayered organisms with their different cell layers separated by basement membranes was dependent on this basement membrane toolkit that has been maintained ever since. Fibrillar collagens are also found in early metazoa, including Hydra and sponges. Interestingly, another collagen, the paralog of collagens XV and XVIII is also ancient, being found in both protostomes and deuterostomes, although the key functions of this class of collagens are not fully understood. Most other collagens are later evolutionary developments, for example the cuticular collagens of Caenorhabditis elegans (Hutter et al. 2000) and the complex collagens with VWA and FN3 domains (see Fig. 1C and Ricard-Blum 2011) found in vertebrates. Also found in all bilateria are the neuronal guidance ECM proteins, netrins, slits, and agrin (Fig. 3).

One characteristic feature of the evolution of ECM proteins, as for other genes, is an increase in numbers of homologous genes as one ascends the tree of life (Fig. 5). Thus, mammals have six type IV collagen genes (see RicardBlum 2011), two nidogen genes, and 11 laminin genes (see Yurchenco 2011) that have arisen by gene duplications and subsequent divergence without altering the basic structures of the proteins. This diversification accompanies the diversification of basement membranes in vertebrates. Similar evolution by duplication and diversification from a primordial gene shared by all bilateria is seen in the case of thrombospondins (see Adams and Lawler 2011), although in this case the diversification has involved more extensive evolution of the domain architecture than is the case for the basement membrane toolkit. This suggests that thrombospondins have evolved to fulfill a more diverse set of functions, whereas basement membranes have retained many of their basic structure-function requirements during the more than half a billion years of their evolution.

Other ECM proteins, in contrast, are more recent developments. Two clear examples are tenascins and fibronectins (Tucker and Chiquet-Ehrismann 2009; Chiquet-Ehrismann and Tucker 2011). Both are restricted to chordates, 
The Matrisome-An Overview of ECM Constituents

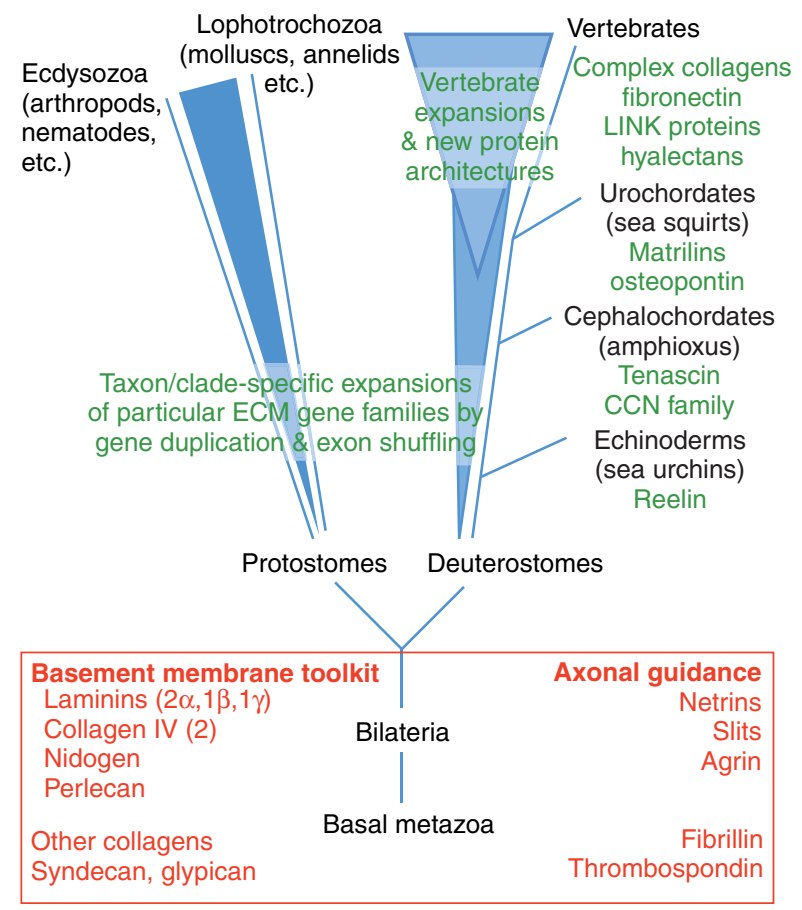

Figure 5. Evolution of ECM proteins. The figure outlines the main phylogenetic lineages (although the branch lengths are not drawn to scale), and illustrates the evolution of complexity of the matrisome and ECM during evolution. The inferred basal bilaterian had a core of ECM proteins including the basement membrane toolkit and some other ECM proteins (not all of which are shown) that have been retained in later-developing taxa, including the two main branches of metazoa (protostomes and deuterostomes). More primitive taxa had some, but not all, of these ECM proteins. During evolution of protostomes, there was modest expansion of the number of ECM genes/proteins mostly comprising taxon-specific expansions of ECM protein families by gene duplication and divergence, with some exon shuffling. A similar modest expansion occurred during evolution of the deuterostome lineage-first known acquisitions of novel ECM proteins of interest are noted in green. During evolution of the vertebrate subphylum, there was a major increase in ECM protein diversity, probably related to two whole genome duplications that occurred in that lineage. This expansion included expansion and diversification of preexisting ECM protein families, and also the development of novel protein architectures by shuffling of domains and the inclusion of novel domains (e.g., FN1, FN2, LINK). Some examples of such novel ECM proteins are indicated. As discussed in the text, this large expansion and diversification of the matrisome in vertebrates is presumably linked to novel structures such as neural crest and endothelial-lined vasculature as well as connective tissues such as cartilage, bones, and teeth, and also the development of more complex nervous and immune systems.

as are many of the more complex collagen genes. A tenascin gene is found in all the chordate genomes that have been sequenced and vertebrates have expanded the tenascin family. Tenascins represent a novel architectural assembly of preexisting domains (EGF and FN3; see Fig. 2). In contrast, fibronectin contains domains that do not appear until quite late in evolution; whereas FN3 domains are ancient, being found in cell-surface receptors in all metazoa, FN1 and FN2 domains are restricted to chordates. The earliest fibronectin-like gene so far reported (although lacking the precise, characteristic domain organization of vertebrate fibronectin) appears in urochordates (ascidians, sea squirts) whereas vertebrates all have the canonical structure found in mammals (see Fig. 2) (Hynes 1990; Schwarzbauer and DeSimone 2011). Once assembled, this gene appears to have been strongly selected (it is 
essential for life) and has remained unchanged. Reelin, a protein that controls aspects of brain development in mammals also appears to be a deuterostome-specific gene (Whittaker et al. 2006), using one old domain (EGF) and two new ones (Reeler and BNR). Analyses of proteoglycans reveal a similar story. Whereas perlecan is ancient (as are the transmembrane proteoglycans, syndecan and glypican), proteoglycans containing the LINK domain are confined to deuterostomes, indeed largely to vertebrates (there are two genes containing that domain in sea urchins) (Whittaker et al. 2006).

In general, it seems clear that the fraction of the proteome that is ECM proteins has expanded disproportionately during the evolution of the deuterostome lineage, both by duplication and divergence of existing genes and by the appearance of novel gene architectures and even some new domains. It is interesting to speculate on the reasons for this. One obvious explanation is the development of cartilage, bones, and teeth in vertebrates and that undoubtedly accounts for some of the elaboration of novel collagens, proteoglycans, and ECM glycoproteins. However, proteins such as tenascins, fibronectin, and reelin (as well as other neural ECM proteins) have no obvious strong connections to the development of structural ECMs and it is tempting to hypothesize that their emergence was more closely tied to the emergence of novel structures such as the neural crest, endothelial-lined vasculature, and more complex nervous systems. Consonant with this model of key roles for ECM proteins in evolution, the matrisome is one of the most plastic and rapidly evolving compartments of the proteome.

\section{CONCLUDING REMARKS}

We now have a reasonably complete inventory of ECM proteins and their associated modifiers. Some ECM proteins have been well studied and we have a good picture of their basic functions-other ECM proteins are virtually unstudied. Even in the case of the well studied proteins, many of the constituent domains, all of which are well conserved and must, therefore, have important functions, still lack assigned functions. Presumably, many of them, like those that we do understand, serve to bind other proteins in ways that contribute to ECM assembly, binding, and presentation of growth factors and interactions with cells to influence their behavior. There is now a pressing need to describe the changes in ECM composition in development and pathology, to better understand the interactions of individual domains, and to probe the cooperation of these multiprotein assemblies in modulating the functions of cells and tissues. The techniques for such analyses (biophysical, imaging, etc.) continue to advance and there is every prospect that studies of ECM structure and function will yield important insights into the crucial roles played by this vital component of metazoan organization, and genetic analyses and studies of human disease are revealing the biological relevance of individual ECM proteins and of specific interactions.

\section{ACKNOWLEDGMENTS}

We thank Charlie Whittaker and Sebastian Hoersch for their assistance and collaboration in the bioinformatic mining of genomes during our development of the ECM inventory discussed here. The work in our laboratory was supported by the National Cancer Institute and the Howard Hughes Medical Institute.

\section{REFERENCES}

Adams J, Engel J. 2007. Bioinformatic analysis of adhesion proteins. In Methods in molecular biology, pp. 147-172. Humana Press, New York.

Adams JC, Lawler J. 2011. The thrombospondins. Cold Spring Harb Perspect Biol doi: 10.1101/cshperspect. a009712.

Apte SS. 2009. A disintegrin-like and metalloprotease (reprolysin-type) with thrombospondin type 1 motif (ADAMTS) superfamily: Functions and mechanisms. J Biol Chem 284: 31493-31497.

Aszódi A, Legate KR, Nakchbandi I, Fässler R. 2006. What mouse mutants teach us about extracellular matrix function. Annu Rev Cell Dev Biol 22: 591-621.

Aumailley M, Bruckner-Tuderman L, Carter WG, Deutzmann R, Edgar D, Ekblom P, Engel J, Engvall E Hohenester E, Jones JCR, et al. 2005. A simplified laminin nomenclature. Matrix Biol 24: 326-332.

Bányai L, Sonderegger P, Patthy L. 2010. Agrin binds BMP2, BMP4 and TGFß1. PLoS ONE 5: e10758. doi: 10.1371/ journal.pone.0010758. 
The Matrisome-An Overview of ECM Constituents

Barresi R, Campbell K. 2006. Dystroglycan: From biosynthesis to pathogenesis of human disease. J Cell Sci 119: 199-207.

Barros CS, Franco SJ, Muller U. 2011. Extracellular matrix: Functions in the nervous system. Cold Spring Harb Perspect Biol 3: a005108.

Bateman JF, Boot-Handford RP, Lamandé SR. 2009. Genetic diseases of connective tissues: Cellular and extracellular effects of ECM mutations. Nat Rev Genet 10: 173-183.

Bentley AA, Adams JC. 2010. The evolution of thrombospondins and their ligand-binding activities. Mol Biol Evol 27: 2187-2197.

Bergmeier W, Hynes RO. 2011. ECM proteins in hemostasis and thrombosis. Cold Spring Harb Perspect Biol doi: 10.1101/cshperspect.a005132.

Bergmeier W, Chauhan AK, Wagner DD. 2008. Glycoprotein Ibalpha and von Willebrand factor in primary platelet adhesion and thrombus formation: Lessons from mutant mice. Thromb Haemost 99: 264-270.

Bishop JR, Schuksz M, Esko JD. 2007. Heparan sulphate proteoglycans fine-tune mammalian physiology. Nature 446: $1030-1037$.

Bix G, Iozzo RV. 2005. Matrix revolutions: "Tails" of basement-membrane components with angiostatic functions. Trends Cell Biol 15: 52-60.

Campbell ID, Humphries MJ. 2011. Integrin structure, activation, and interactions. Cold Spring Harb Perspect Biol 3: a004994.

Cawston TE, Young DA. 2010. Proteinases involved in matrix turnover during cartilage and bone breakdown. Cell Tissue Res 339: 221-235.

Chapman JA, Kirkness EF, Simakov O, Hampson SE, Mitros T, Weinmaier T, Rattei T, Balasubramanian PG, Borman J, Busam D, et al. 2010. The dynamic genome of Hydra. Nature 464: 592-596.

Chautard E, Ballut L, Thierry-Mieg N, Ricard-Blum S. 2009. MatrixDB, a database focused on extracellular protein-protein and protein-carbohydrate interactions. Bioinformatics 25: 690-691.

Chautard E, Fatoux-Ardore M, Ballut L, Thierry-Mieg N, Ricard-Blum S. 2010. MatrixDB, the extracellular matrix interaction database. Nucleic Acids Res 39: D235-D240.

Chen C-C, Lau LF. 2009. Functions and mechanisms of action of CCN matricellular proteins. Int J Biochem Cell Biol 41: 771-783.

Chiquet-Ehrismann R, Tucker RP. 2011. Tenascins and the importance of adhesion modulation. Cold Spring Harb Perspect Biol 3: a004960.

Choi MG, Hynes RO. 1979. Biosynthesis and processing of fibronectin in NIL.8 hamster cells. J Biol Chem 254: 12050-12055.

Couchman JR. 2010. Transmembrane signaling proteoglycans. Annu Rev Cell Dev Biol 26: 89-114.

Daley WP, Peters SB, Larsen M. 2008. Extracellular matrix dynamics in development and regenerative medicine. J Cell Sci 121: 255-264.

de Vega S, Iwamoto T, Yamada Y. 2009. Fibulins: Multiple roles in matrix structures and tissue functions. Cell Mol Life Sci 66: 1890-1902.
Engel J. 1996. Domain organizations of modular extracellular matrix proteins and their evolution. Matrix Biol 15: 295-299.

Eyre DR, Wu J.-J. 2005. Collagen cross-links. In Topics in current chemistry, Vol. 247, pp. 207-229. SpringerVerlag, Berlin.

Filmus J, Capurro M, Rast J. 2008. Glypicans. Genome Biol 9: 224.

Geiger B, Yamada KM. 2011. Molecular architecture and function of matrix adhesions. Cold Spring Harb Perspect Biol 3: a005033.

Gordon MK, Hahn RA. 2009. Collagens. Cell Tissue Res 339: $247-257$.

Hay ED, ed. 1981. Cell biology of extracellular matrix. Plenum Press, New York.

Hay ED, ed. 1991. Cell biology of extracellular matrix, 2nd ed. Plenum Press, New York.

Ho MSP, Böse K, Mokkapati S, Nischt R, Smyth N. 2008. Nidogens_Extracellular matrix linker molecules. Microsc Res Tech 71: 387-395.

Hohenester E, Engel J. 2002. Domain structure and organisation in extracellular matrix proteins. Matrix Biol 21: 115-128.

Humphries JD, Byron A, Humphries MJ. 2006. Integrin ligands at a glance. J Cell Sci 119: 3901-3903.

Huttenlocher A, Horwitz A. 2011. Integrins in cell migration. Cold Spring Harb Perspect Biol doi: 10.1101/ cshperspect.a005074.

Hutter H, Vogel BE, Plenefisch JD, Norris CR, Proenca RB, Spieth J, Guo C, Mastwal S, Zhu X, Scheel J, et al. 2000. Conservation and novelty in the evolution of cell adhesion and extracellular matrix genes. Science 287: 989-994.

Huxley-Jones J, Robertson DL, Boot-Handford RP. 2007. On the origins of the extracellular matrix in vertebrates. Matrix Biol 26: 2-11.

Hynes RO. 1990. Fibronectins. Springer-Verlag, New York.

Hynes RO. 2002. Integrins: Bidirectional, allosteric signaling machines. Cell 110: 673-687.

Hynes RO. 2007. Cell-matrix adhesion in vascular development. J Thromb Haemost 5 (Suppl 1): 32-40.

Hynes RO. 2009. The extracellular matrix: Not just pretty fibrils. Science 326: 1216-1219.

Hynes RO, Zhao Q. 2000. The evolution of cell adhesion. J Cell Biol 150: F89-F96.

Iismaa SE, Mearns BM, Lorand L, Graham RM. 2009. Transglutaminases and disease: Lessons from genetically engineered mouse models and inherited disorders. Physiol Rev 89: 991-1023.

Iozzo RV, Murdoch AD. 1996. Proteoglycans of the extracellular environment: Clues from the gene and protein side offer novel perspectives in molecular diversity and function. FASEB J 10: 598-614.

Kessenbrock K, Plaks V, Werb Z. 2010. Matrix metalloproteinases: Regulators of the tumor microenvironment. Cell 141: 52-67.

Leitinger B, Hohenester E. 2007. Mammalian collagen receptors. Matrix Biol 26: 146-155.

Lin F, Ren X-D, Pan Z, Macri L, Zong W-X, Tonnesen MG, Rafailovich M, Bar-Sagi D, Clark RAF. 2010. Fibronectin 
growth factor-binding domains are required for fibroblast survival. J Invest Dermatol 131: 84-98.

Lorand L, Graham R. 2003. Transglutaminases: Crosslinking enzymes with pleiotropic functions. Nat Rev Mol Cell Biol 4: 140-156.

Lu P, Takai P, Weaver VM, Werb Z. 2011. Extracellular matrix degradation and remodeling in development and disease. Cold Spring Harb Perspect Biol doi: 10.1101/cshperspect.a005058.

Mecham R. 2011. The extracellular matrix: An overview. Springer, Berlin.

Merline R, Schaefer R, Schaefer L. 2009. The matricellular functions of small leucine- rich proteoglycans (SLRPs). J. Cell Commun Signal 3: 323-335.

Mott JD, Werb Z. 2004. Regulation of matrix biology by matrix metalloproteinases. Curr Opin Cell Biol 16: $558-564$.

Munger JS, Sheppard D. 2011. Crosstalk among TGF $\beta$ signaling pathways, integrins, and the extracellular matrix Cold Spring Harb Perspect Biol doi: 10.1101/ cshperspect.a005017.

Murphy G. 2008. The ADAMs: Signalling scissors in the tumour microenvironment. Nat Rev Cancer 8: 929-941.

Naba A, Clauser KR, Hoersch S, Liu H, Carr SA, Hynes RO. 2011. The matrisome: In silico definition and in vivo characterization by proteomics of normal and tumor extracellular matrices (in revision).

Nelson CM, Bissell MJ. 2006. Of extracellular matrix, scaffolds, and signaling: Tissue architecture regulates development, homeostasis, and cancer. Annu Rev Cell Dev Biol 22: 287-309.

Nyberg P, Xie L, Kalluri R. 2005. Endogenous inhibitors of angiogenesis. Cancer Res 65: 3967-3979.

Ozbek S, Balasubramanian PG, Chiquet-Ehrismann R, Tucker RP, Adams JC. 2010. The evolution of extracellular matrix. Mol Biol Cell 21: 4300-4305.

Page-McCaw A, Ewald AJ, Werb Z. 2007. Matrix metalloproteinases and the regulation of tissue remodelling. Nat Rev Mol Cell Biol 8: 221-233.

Patthy L. 1999. Genome evolution and the evolution of exon-shuffling-A review. Gene 238: 103-114.

Pontén F, Jirström K, Uhlen M. 2008. The human protein atlas-A tool for pathology. J Pathol 216: 387-393.

Porter S, Clark IM, Kevorkian L, Edwards DR. 2005. The ADAMTS metalloproteinases. Biochem J 386: 15-27.

Putnam N, Srivastava M, Hellsten U, Dirks B, Chapman J, Salamov A, Terry A, Shapiro H, Lindquist E, Kapitonov VV, et al. 2007. Sea anemone genome reveals ancestral eumetazoan gene repertoire and genomic organization. Science 317: 86-94.

Rahman S, Patel Y, Murray J, Patel KV, Sumathipala R, Sobel M, Wijelath ES. 2005. Novel hepatocyte growth factor (HGF) binding domains on fibronectin and vitronectin coordinate a distinct and amplified Met-integrin induced signalling pathway in endothelial cells. BMC Cell Biol 6: 8 .

Ramirez F, Dietz HC. 2009. Extracellular microfibrils in vertebrate development and disease processes. J Biol Chem 284: $14677-14681$.
Ramirez F, Rifkin DB. 2009. Extracellular microfibrils: Contextual platforms for TGF $\beta$ and BMP signaling. Curr Opin Cell Biol 21: 616-622.

Ricard-Blum S. 2011. The collagen family. Cold Spring Harb Perspect Biol 3: a004978.

Robins SP. 2007. Biochemistry and functional significance of collagen cross-linking. Biochem Soc Trans 35: 849-852.

Rozario T, DeSimone D. 2010. The extracellular matrix in development and morphogenesis: A dynamic view. Dev Biol 341: 126-140.

Sarrazin S, Lamanna WC, Esko JD. 2011. Heparan sulfate proteoglycans. Cold Spring Harb Perspect Biol 3: a004952.

Schaefer L, Schaefer RM. 2010. Proteoglycans: From structural compounds to signaling molecules. Cell Tissue Res 339: $237-246$.

Schwartz MA. 2010. Integrins and extracellular matrix in mechanotransduction. Cold Spring Harb Perspect Biol 2: a005066.

Schwarzbauer JE, DeSimone DW. 2011. Fibronectins, their fibrillogenesis and in vivo functions. Cold Spring Harb Perspect Biol 3: a005041.

Srivastava M, Simakov O, Chapman J, Fahey B, Gauthier MEA, Mitros T, Richards GS, Conaco C, Dacre M, Hellsten U, et al. 2010. The Amphimedon queenslandica genome and the evolution of animal complexity. Nature 466: $720-726$.

Tucker RP, Chiquet-Ehrismann R. 2009. Evidence for the evolution of tenascin and fibronectin early in the chordate lineage. Int J Biochem Cell Biol 41: 424-434.

Uhlen M, Oksvold P, Fagerberg L, Lundberg E, Jonasson K, Forsberg M, Zwahlen M, Kampf C, Wester K, Hober S, et al. 2010. Towards a knowledge-based human protein atlas. Nat Biotechnol 28: 1248-1250.

Wang X, Harris RE, Bayston LJ, Ashe HL. 2008. Type IV collagens regulate BMP signalling in Drosophila. Nature 455: 72-77.

Watt FM, Fujiwara H. 2011. Cell-extracellular matrix interactions in normal and diseased skin. Cold Spring Harb Perspect Biol 3: a005124.

Whittaker CA, Bergeron K-F, Whittle J, Brandhorst BP, Burke RD, Hynes RO. 2006. The echinoderm adhesome Dev Biol 300: 252-266.

Wickström SA, Radovanac K, Fässler R. 2011. Genetic analyses of integrin signaling. Cold Spring Harb Perspect Biol 3: a005116.

Wijelath ES, Rahman S, Namekata M, Murray J, Nishimura T, Mostafavi-Pour Z, Patel Y, Suda Y, Humphries MJ, Sobel M. 2006. Heparin-II Domain of fibronectin is a vascular endothelial growth factor-binding domain: Enhancement of VEGF biological activity by a singular growth factor/matrix protein synergism. Circ Res 99: 853-860.

Xian X, Gopal S, Couchman JR. 2010. Syndecans as receptors and organizers of the extracellular matrix. Cell Tissue Res 339: 31-46.

Yan D, Lin X. 2009. Shaping morphogen gradients by proteoglycans. Cold Spring Harb Perspect Biol 1: a002493.

Yurchenco PD. 2011. Basement membranes: Cell scaffoldings and signaling platforms. Cold Spring Harb Perspect Biol 3: a004911. 


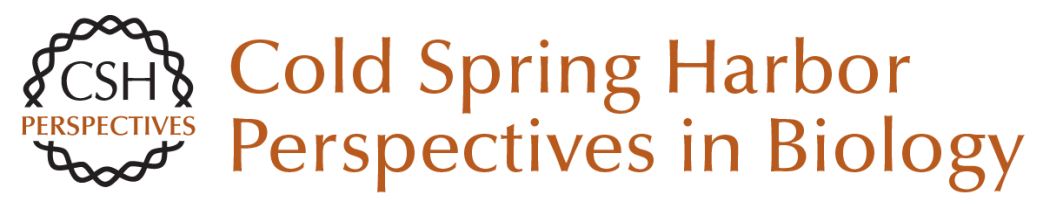

\section{Overview of the Matrisome--An Inventory of Extracellular Matrix Constituents and Functions}

Richard O. Hynes and Alexandra Naba

Cold Spring Harb Perspect Biol 2012; doi: 10.1101/cshperspect.a004903 originally published online September 21, 2011

Subject Collection Extracellular Matrix Biology

Extracellular Matrix in Development: Insights from Mechanisms Conserved between Invertebrates and Vertebrates Nicholas H. Brown

Extracellular Matrix Proteins in Hemostasis and Thrombosis Wolfgang Bergmeier and Richard O. Hynes

The Thrombospondins Josephine C. Adams and Jack Lawler

Cross Talk among TGF- $\beta$ Signaling Pathways, Integrins, and the Extracellular Matrix John S. Munger and Dean Sheppard

Heparan Sulfate Proteoglycans Stephane Sarrazin, William C. Lamanna and Jeffrey D. Esko

The Collagen Family Sylvie Ricard-Blum

Tenascins and the Importance of Adhesion Modulation Ruth Chiquet-Ehrismann and Richard P. Tucker Integrin Structure, Activation, and Interactions lain D. Campbell and Martin J. Humphries
Extracellular Matrix Degradation and Remodeling in Development and Disease

Pengfei Lu, Ken Takai, Valerie M. Weaver, et al.

Overview of the Matrisome--An Inventory of

Extracellular Matrix Constituents and Functions Richard O. Hynes and Alexandra Naba

Integrins in Cell Migration Anna Huttenlocher and Alan Rick Horwitz

Fibronectins, Their Fibrillogenesis, and In Vivo Functions Jean E. Schwarzbauer and Douglas W. DeSimone

Extracellular Matrix: Functions in the Nervous System

Claudia S. Barros, Santos J. Franco and Ulrich Müller

Molecular Architecture and Function of Matrix Adhesions Benjamin Geiger and Kenneth M. Yamada

Cell-Extracellular Matrix Interactions in Normal and Diseased Skin

Fiona M. Watt and Hironobu Fujiwara

Genetic Analyses of Integrin Signaling

Sara A. Wickström, Korana Radovanac and Reinhard Fässler

For additional articles in this collection, see http://cshperspectives.cshlp.org/cgi/collection/

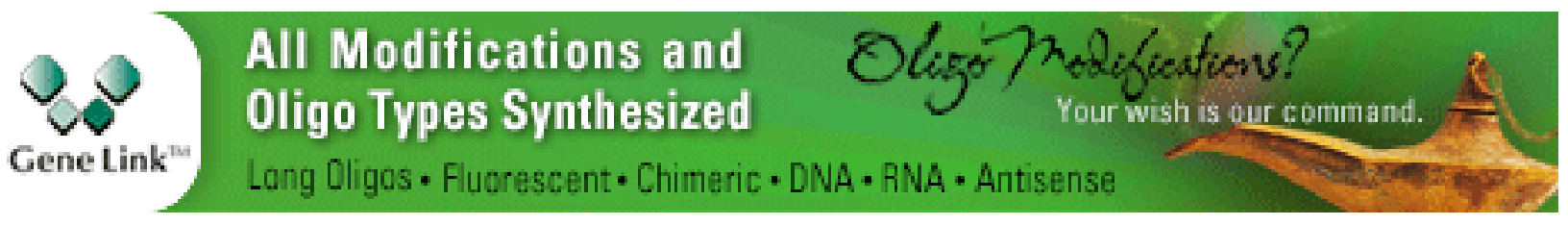

Copyright @ 2012 Cold Spring Harbor Laboratory Press; all rights reserved 